\title{
WIR haben unsere Hausaufgaben gemacht
}

\author{
oder: Wie der Zweck denselbigen erreicht hat ...
}

Jean-Pierre Pochon, Mirjam Buchmann, Nicola Moser

Korrespondenz:

PD Dr. med. Jean-Pierre Pochon Tagesklinik für Kinderchirurgie Dübendorfstrasse 20

CH-8117 Fällanden

pochon@ambikid.ch

www.tokterhuus.ch

\section{Wer sind WIR?}

Die Tagesklinik für Kinderchirurgie in Fällanden ZH besteht seit 1989. Aktuell sind drei Ärztinnen bzw. Ärzte und 13 Mitarbeiter/innen darin beschäftigt. Unser Tätigkeitsgebiet umfasst die gesamte Kinderchirurgie inklusive Traumatologie, sofern die Eingriffe ambulant durchführbar sind.

Etwa zwei Drittel der jährlich rund 1700 Operationen erfolgten in Allgemeinnarkose, meist kombiniert mit peripheren Nervenblöcken, ein Drittel in Lokalanästhesie. Wir verfügen über einen anerkannten OP I und einen Praxis-OP, zwei Aufwachräume mit sieben Betten, die mit Überwachungsmonitoren und Sauerstoffanschlüssen ausgerüstet sind.

Alle Eingriffe werden nach dem jeweils gültigen Allgemeintarif durchgeführt.

Die Grösse unserer Klinik erlaubt es, sehr differenzierte, echt (!) betriebswirtschaftliche Analysen sämtlicher Leistungen und Fixkosten in einem ambulanten chirurgischen Betrieb aufzuzeigen. Damit gelten die Angaben im folgenden auch für die ambulante Chirurgie bei Erwachsenen, ja für ein Spitalambulatorium, soweit dessen Kennzahlen nicht in den allgemeinen Spitalbetrieb einfliessen.

Wir beschränken uns im folgenden auf die betriebswirtschaftlichen Kennzahlen im Zeitraum der Jahre 2002 bis und mit 2005, die auf den Erträgen der Sprechstunden und der bei uns durchgeführten ambulanten Eingriffen basieren.

\section{Von GRAT ZU TARMED - oder «la grande illusion» ...}

Sie erinnern sich: Im Jahr 2002 wurden unsere ambulanten Leistungen nach dem Zürcher Krankenkassentarif und nach dem gesamtschweizerischen («blauen») IV-Tarif abgerechnet. Der Taxpunktwert im Kt. Zürich betrug Fr. -.80 für die Krankenkassenpatienten («KK») und Fr. 4.95 für die IV-Patienten.

Per 1. Mai 2003 wurde für die UV/MV/IVPatienten TARMED mit einem Starttaxpunktwert von Fr. 1.- eingeführt. Am 1. Januar 2004 folgte der TARMED-Tarif für die KK-Patienten mit einem Starttaxpunktwert von Fr. -.97.

Dass der TPW bereits im Vorfeld zwischen der Beta-3- und der Alpha-2-Version um 17\%

\section{NOUS avons fait nos devoirs au-delà de toute attente ...}

Suite à une nouvelle réduction des valeurs du point tarifaire imposée dans le canton de Zurich, l'auteur compare la situation économique d'un service de chirurgie pédiatrique ambulatoire avant et après l'introduction du TARMED. Un plan comptable différencié lui permet désormais de détecter et de justifier les répercussions négatives induites par les nouveaux tarifs dans le domaine des caisses-maladie et de l'assuranceinvalidité. Grosso modo, l'expérience faite dans une PME de chirurgie vaut également pour d'autres centres chirurgicaux ambulatoires recevant des adultes et qui doivent s'en sortir avec le tarif général, mais aussi pour les cabinets de groupe prodiguant des soins de premier recours.

Bien que les instances supérieures l'aient toujours douté, le bénéfice du bilan, considéré sur une base de $100 \%$ en 2002 , a diminué à $72,4 \%$ en 2005 . En revanche, la part salariale du personnel augmente en comparaison, malgré la réduction des autres coûts d'exploitation.

Cet effet, qui se développe le plus souvent de manière furtive, est connu depuis longtemps dans les entreprises de production et de services. Il a frappé le corps médical de plein fouet à la suite du TARMED, notamment par les différentes réductions de la valeur du point tarifaire pour des raisons compensatoires. L'auteur décrit plusieurs situations en matière d'économie d'entreprise et propose quelques solutions susceptibles de contrer cette évolution en la régulant. 


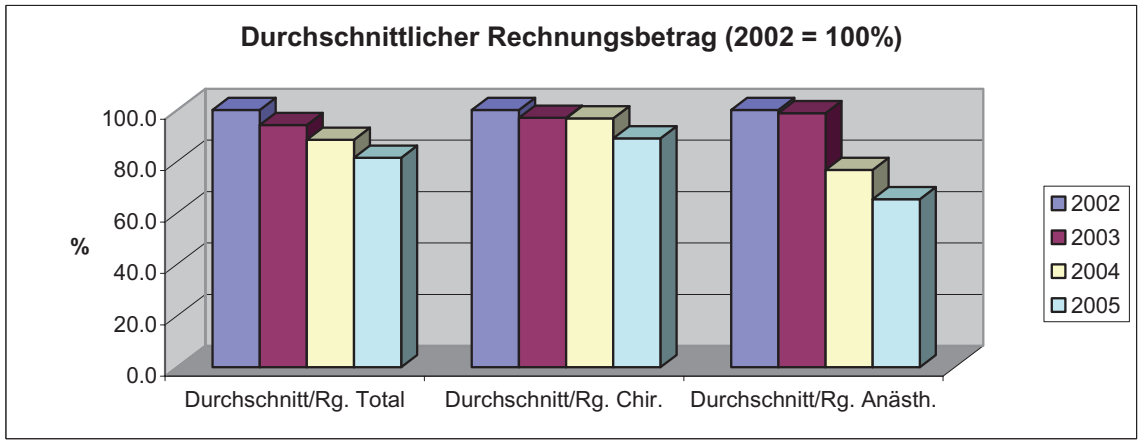

Abbildung 1

Auswirkungen von TARMED auf die durchschnittliche Höhe unserer Rechnungen (Sprechstunde und Operationen).

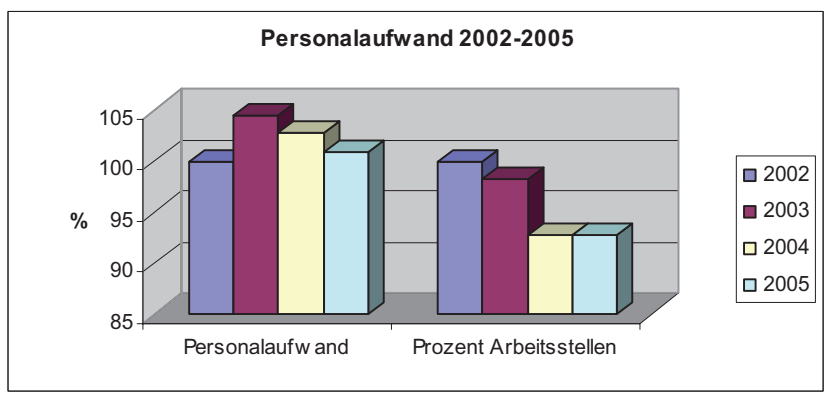

Abbildung 2

Stellenprozente und Entwicklung der Personalkosten.

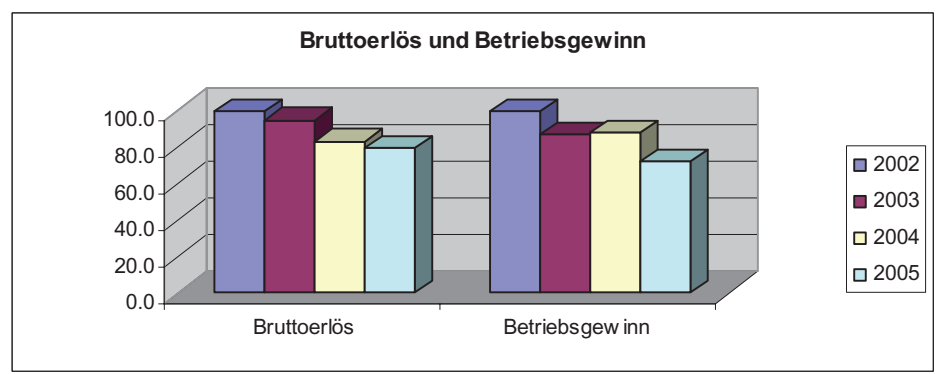

Abbildung 3

Bruttoerlös im Vergleich zum Betriebsgewinn.

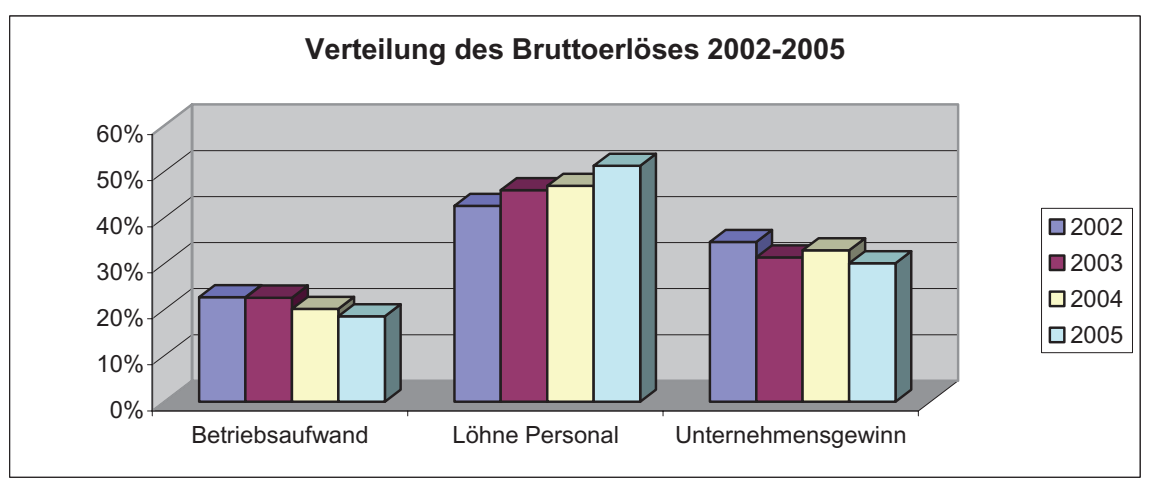

Abbildung 4

Prozentuale Aufteilung des Bruttoerlöses auf Betriebsaufwand, Personallöhne

(inkl. gesetzlicher Abgaben) und Unternehmens- oder Betriebsgewinn. gesenkt wurde [2], liess Böses ahnen, störte aber offenbar die Pro-TARMED-Stimmenden nicht.

Durch die unselige Kostenneutralität kamen die TPW weiter unter Druck, im Kanton Zürich erfolgte bereits am 1. September 2004 bei den KK-Patienten eine Reduktion auf Fr. -.95, am 1. Februar 2005 auf Fr. -.91 und am 1. Juli 2005 gar auf Fr. -.90 [3]. Ebenso fiel der TPW bei den IV-Patienten auf Fr. -.92! In unserem Fall ist es besonders stossend, dass die gleiche ambulante Tätigkeit an einem Spital immer noch mit Fr. 1.- abgegolten wird, obwohl die Infrastrukturkosten und die Ausrüstung dieselben sind.

\section{Die chirurgische «technische» Leistung ist tot, es lebe die «intellektuelle»?}

Durch unsere früheren Warenkorbstudien war eine erhebliche Einbusse unserer - wohlverstanden nur mit Allgemeinpatienten erwirtschafteten - Honorare zu erwarten.

Auch Vertreter der damaligen Zürcher FMS (unter anderem der Schreibende) konnten Herrn Brunner von der Problematik, dass die erhebliche Verminderung der ärztlichen (chirurgischen) Leistung zum Beispiel auch das in kleinen Spitälern bestens funktionierende Belegarztsystem gefährdet, nicht überzeugen.

Das Beispiel der Leistenhernie beim Kind ein typischer ambulanter kinderchirurgischer Eingriff - wurde nicht ernst genommen, obwohl die in Tabelle 1 (Seite 1600) angeführten Zahlen real und den von den Versicherern akzeptierten Honorarrechnungen entsprachen. Im Gegenteil, der betriebswirtschaftliche Hintergrund einer Operation, allein die Betriebskosten in der Höhe von über $65 \%$ des Umsatzes, wurden schlicht bezweifelt.

In der Zeit zwischen dem Allgemeintarif im Jahre 2002 und dem sukzessiv eingeführten TARMED-Tarif bis Ende 2005 verminderte sich die Höhe der Rechnungen, trotz vergleichbarem Krankengut und vergleichbarer Auslastung.

Die durchschnittliche Höhe der Rechnungen reduzierte sich - auf die gesamte Klinik bezogen - im Jahre 2005 auf 81,4\% gegenüber 2002. Die kinderchirurgischen Rechnungen sanken auf $92,2 \%$, die anästhesiologischen gar auf $65,7 \%$ gegenüber 2002 (Abb. 1).

Die Betriebskosten sind effizient nur auf dem Niveau Personalkosten zu beeinflussen. Trotz Verringerung der Beschäftigungsgrade im Jahr 2004 auf 92\% stiegen die Lohnkosten inklusive AHV/ALV/BVG-Abgaben im Jahre 2003 im Vergleich zu 2002 auf 104,5\%. Ein Trend zur Verminderung zeichnete sich durch die Reduktion der OP-Tage auf vier Tage pro Woche und 
die damit verbundene Senkung des Beschäftigungsgrades einiger Angestellter auf 80\% erst im Jahre $2004 \mathrm{ab}$. Die Personalkosten liegen im Jahre 2005 mit 100,8\% noch knapp über dem Ausgangswert des Jahres 2002 (Abb. 2).

Im Jahr 2005 sank der Bruttoerlös (Summe der effektiv erzielten Honorare) gegenüber 2002 auf $79,6 \%$, der Betriebsgewinn gar auf 72,4\% (Abb. 3), obwohl alle irgend möglichen Einsparungen gemacht wurden. Der Betriebsgewinn im Jahr 2005 sank damit um rund 7\% mehr als der Bruttoerlös.

Interessant ist die Verteilung des Bruttoerlöses prozentual zum Betriebsaufwand, zu den Personallöhnen und dem den Unternehmern verbleibenden Gewinn. Während der Betriebsaufwand von $23 \%$ auf $19 \%$ gesenkt werden konnte, stieg der prozentuale Anteil der Löhne wegen der wenig wirksamen Personalkostenreduktion von $43 \%$ auf 51\% des Erlöses. Entsprechend verringerte sich der Unternehmensgewinn von 35\% auf unter 30\% (Abb. 4).

\section{Analyse und Gegenmassnahmen, oder: «Die Senftube ist ausgedrückt»}

Es würde den Rahmen sprengen, auf alle Detailstatistiken einzugehen. Fest steht, dass die durch TARMED verursachten Einbussen die untere Toleranzgrenze in einem allgemeinchirurgischen Unternehmen längstens erreicht haben.

Am Beispiel der «Herniotomie beim Kind unter sieben Jahren» fällt die Mindereinnahme von über 30\% auf. Zu Zeiten des «blauen» Tarifs konnten wir mit einem anerkannten OP I die Nebenkosten mit 85\% des Arzthonorars verrechnen. Selbst wenn nach TARMED die Position 35.0030, «technische Grundleistung OP I anerkannt», dazu verrechnet wird (TP 179.65 = Fr. 165.28), werden wir immer noch um Fr. 353.40 oder $30,7 \%$ weniger entschädigt.

Man wirft uns vor, bei diesen Beispielen nicht die einzelnen Positionen zu vergleichen. Das stimmt. Aber für unseren Betrieb interessiert es nicht, ob einzelne Positionen eventuell sogar besser entschädigt werden. Uns interessiert betriebswirtschaftlich, was pro Fall oder pro Behandlung - salopp ausgedrückt - unter dem Strich nach Abzug aller Unkosten übrigbleibt.

Dass die Differenz der chirurgischen Rechnungsbeträge im Zeitraum 2002-2005 mit rund 8 Prozent im Endeffekt relativ knapp ausfällt, erstaunt nicht: Häufige, «einfachere» Operationen werden deutlich unterhonoriert. Sie können durch seltenere, «schwierigere» Eingriffe durch deren bessere Honorierung kompensiert werden. Wer keine solche Eingriffe durchführen kann oder will, ist jedoch der Bestrafte! Diese Art von
Quersubventionierung entbehrt jeder Logik und ist die Folge der arithmetischen BlackBox-Rechnerei in der Zeit zwischen «GRAT» und «TARMED»!

Anlässlich eines Gesprächs mit Vertretern der IV, Vertretern des Tarifdienstes FMH, Herrn Kollegen Tönz sowie dem Schreibenden wurde unseren Zahlen erwartungsgemäss mittels einer IV-internen Statistik widersprochen: In der Tat variieren die Kosten der Kinderchirurgen nicht wesentlich von den der IV in Rechnung gestellten Honorare der anderen Leistungserbringer.

Warum? Die IV erfasst nur die von den Leistungserbringern direkt und unter ihrem Namen in Rechnung gestellten Leistungen. Ausser an unserer Tagesklinik werden alle Operationen der Geburtsgebrechenliste belegärztlich an Kliniken durchgeführt, von diesen in Rechnung gestellt und unter den Klinikleistungen subsumiert! Diese Leistungen erscheinen nicht namentlich unter z.B. der EAN-Nummer des entsprechenden Kinderchirurgen. Die IV-Statistik erfasst somit nur die Sprechstundenleistungen in der Praxis, die logischerweise bei allen Fachärztinnen und -ärzten etwa gleich hoch sind, was ein falsches, die Operationen nicht erfassendes Bild ergibt.

Dass der Bruttoerlös aus Sprechstundentätigkeit und Operationen etwa parallel mit den durchschnittlichen Rechnungsbeträgen abfällt, ist bei gleichem Krankengut und gleicher Auslastung eine logische Konsequenz. Hingegen lässt die deutliche Minderhonorierung der kinderanästhesiologischen Leistungen im Vergleich $\mathrm{zu}$ den chirurgischen mehr als aufhorchen, sanken doch die Anästhesieerträge um mehr als ein Drittel auf 65,7\%! Für den Erhalt eines chirurgischen Ambulatoriums müsste notgedrungen die Anästhesiologie durch die Chirurgie quersubventioniert werden, was doch kaum einem modernen Abgeltungssystem (vor allem bei voneinander unabhängigen Geschäftspartnern) entsprechen dürfte! Chirurgie- und Anästhesiezeiten sowie Risikoklassen müssten endlich wieder entkoppelt werden, kann doch eine chirurgisch banale Operation bei einem anästhesiologisch heiklen Patienten nicht über den gleichen Leisten geschlagen werden!

Die Betriebskosten setzen sich zum grössten Teil aus den Personalkosten zusammen - zurzeit etwa 1 Million Franken (!) -, obwohl wir keinesfalls Spitzenlöhne ausrichten. In einem kleinen chirurgischen Team sind einer Reduktion des Personalbestandes sowohl qualitativ wie auch quantitativ Grenzen gesetzt.

In den höchsten Tönen wird uns das Hohelied der Qualitätskontrolle und der Qualitätssicherung vorgesungen. Diese hängt zu einem 
grossen Teil direkt mit dem Ausbildungsstand und der Selbständigkeit des Personals zusammen. Wie diese Qualität als Standard unter dem finanziellen Druck der hohen Löhne und Versicherungsabgaben gehalten werden soll, wird geflissentlich verschwiegen.

Bei 48 Wochen Praxisbetrieb und durchschnittlich 5 Wochen Ferien pro Angestellte fehlt bei 12 Mitarbeitern/-innen jede Woche mindestens eine. Entsprechend muss mehr und polyvalentes Personal für den Ersatz bei Ferien und Krankheit eingestellt werden. Erfahrene ältere Mitarbeiter/innen haben Anspruch auf eine höhere Entlöhnung, fallen aber auch in die Gruppe der höchsten BVG-Abgaben. In einem KMU im Gesundheitswesen können nicht wie in einer staatlich subventionierten Klinik als Ausgleich auszubildende Pflegefachfrauen oder Unterassistenten beschäftigt werden, um so mehr als wir im konkreten Fall als Ausbildungsstätte gar nicht anerkannt sind.

Wenig überraschend aus betriebswirtschaftlicher Sicht, jedoch kritisch für den Erhalt eines Unternehmens ist die Tatsache, dass der prozentuale Anteil der Angestelltenlöhne steigt (bzw. nominal etwa gleich bleibt), der Lohn des Unternehmers jedoch sinkt, wenn der Bruttoerlös abnimmt. Wir haben bewiesen, dass es wohl gelingt, durch Rationalisierung der Abläufe im Betrieb, Straffen des Personaleinsatzes und günstige Materialbeschaffungskosten, Rückzahlungen der Kredite und damit Zinseinsparungen die Betriebskosten um bis zu 30\% zu senken. In vielen Praxen ist dies jedoch bereits erfolgt, so dass sich weitere Taxpunktwertsenkungen noch extremer auswirken.

Wie unethisch ist es also, in der Medizin von Geld zu sprechen? Scheitern wir wieder einmal mehr an der Frage, wieviel eine Ärztin oder ein Arzt brutto, vor Abzug Altersvorsorge verdienen darf? Wurden wirklich die hohen Investitionsund Betriebskosten in gut eingerichteten Praxen von Grundversorgern oder Spezialärzten/-ärztinnen in der sogenannt «betriebswirtschaftlichen» Berechnung der TL miteinberechnet?

Von einem herzhaften, dankbaren Händedruck des geheilten Patienten lassen sich leider keine Investitionskredite, keine Mietzinsen und keine Löhne bezahlen ...

Wo also ansetzen? Bei den nicht beeinflussbaren Positionen? Wohl kaum! Etwa:

- Bei den berüchtigten, dem Patienten nicht verrechenbaren Materialien, bei Hypozinsen oder indexgebundenen Mietzinsen?

- Bei Heizungs-, Strom- und Benzinkosten?

- Beim Einkauf? Durch günstige Einkaufsbedingungen können Lieferanten aus der Pharma- und Hilfsmittelindustrie gegebenenfalls entgegenkommen, wobei auch hier immer mehr Grenzen gesetzt sind.

- Bei den Taxpunktwerten? Sollen die Bündner in den Jura umziehen, weil dort 100 TP 99 Franken, in Graubünden nur 78 Franken ergeben?

- Bei den solidarisch zu bezahlenden Gebühren für die Hygienekontrollen im OP I, auch wenn niemand im betreffenden Jahr kontrolliert hat?

- Bei den Kosten für die periodischen Kontrollen der Röntgenanlage?

Vielleicht bei den nur bedingt beeinflussbaren Positionen?

- Bei den Löhnen? Die Löhne für innovative, selbständige Mitarbeiter/innen sind, wenn nur einigermassen mit den an den Kliniken ausgerichteten Löhnen mitgehalten werden soll, relativ hoch. Sie werden wenigstens durch motivierten Einsatz mehr als wettgemacht. (Nicht nur Patienteneltern staunen, wenn wir von unseren Lohnkosten im Bereich von einer Million Franken sprechen.)

- Beim Angebot? Das medizinische Angebot und die Anzahl der OP-Tage könnten reduziert, die Arbeitsintensität dagegen auf Kosten der Patientenbetreuung (?!) gesteigert werden. Damit würden nur noch Angestellte im Teilzeitbereich zwischen 50 und 70\% beschäftigt. Für unsere zum Teil langjährigen Mitarbeiter/innen bedeutet dies Aufgabe ihrer Stelle und Suche nach Vollbeschäftigung. Dass dies für Arbeitnehmer/innen im Alter über 50 Jahre praktisch erfolglos ist, liegt auf der Hand.

- Bei der Personalsuche? Sollen also nur noch junge, «BVG-freundliche» Mitarbeiter/innen eingestellt oder soll im Ausland nach billigeren Kräften gesucht werden?

- Bei Trendmedizin? Sollen wir auf cash zu bezahlende Gesundheitsberatungen usw. umstellen und kosmetische Nichtpflichtleistungen anbieten?

Bei den beeinflussbaren Positionen?

- Bei Finanzoptimierungen oder bei der Rechtsform der Praxis? Das Modell der Ärzte-AG, wie von Herrn Reichen (Visana) am «ZischtigsClub» vom 5. April 2006 vorgeschlagen, scheitert im Kanton Zürich an den kantonalen Gesetzen, auch wenn damit gewisse Optimierungen erreicht werden könnten.

- Bei Gruppenpraxen/Teilzeitmitarbeitern/-innen? Irgendwann lässt sich der Praxisaufwand durch Verteilung auf weitere Praxis- 
kollegen/-innen nicht mehr senken. Der resultierende Mehrgewinn fällt nach dem Aufteilen unter eine realistische Grösse für den Erhalt einer Familie mit Kindern, es sei denn, das Einkommen «teilt» sich ein Ärzteehepaar.

\section{Zukunftsperspektiven und ein paar verrückte Zahlenübungen ...}

Gehen wir von den von Niklaus Hasler publizierten durchschnittlichen steuerbaren Ein-

\section{Tabelle 1}

Vergleich der IV-Honorierung einer Leistenhernie, einseitig, beim Knaben unter 7 Jahren zum alten «blauen Tarif» und TARMED 2005, TPW à 0,92. Der ärztliche Assistenzanteil beträgt dabei 100,01 TP $=$ Fr. 92.01, das heisst Fr. 72.99 weniger als bisher.

Herniotomie einseitig, Knabe, 5 Jahre alt, inkl. ärztlicher Assistenz.

OP-Tag inkl. Nebenkosten.

\section{IV-Tarif (Blauer Tarif)}

TPW: 4,95

\begin{tabular}{lllcc} 
Anzahl & Position & Text & Taxpunkte & Fr. \\
\hline 1 & 1015 & Erste Konsultation & 8 & 39.60 \\
1 & 1073 & Vermehrter Zeitaufwand & 6 & 29.70 \\
1 & 2402 & Leistenhernie einseitig & 100 & $495 .-$ \\
1 & 3501 & Ärztl. Assistenz ( $1 / 3$ OP-Honorar) & 33,3 & $165 .-$ \\
1 & $1902 / 03$ & Nebenkosten $85 \%$ OP-Honorar & 85 & 420.75 \\
TOTAL & & & & $\mathbf{1 1 5 0 . 0 5}$
\end{tabular}

\section{TARMED}

TPW: 0,92

OP-Tag AL+TL inkl. Assistenz.

\begin{tabular}{lrlrr}
\hline Anzahl & Position & Text & Taxpunkte & \multicolumn{1}{c}{ Fr. } \\
\hline 1 & 00.0010 & Konsultation & 17,76 & 16.34 \\
\hline 2 & 00.0020 & weitere 5' & 17,76 & 32.68 \\
\hline 1 & 00.0030 & letzte 5' & 8,88 & 8.17 \\
\hline 1 & 00.0040 & Kind unter 6 Jahren & 13,11 & 12.06 \\
\hline 1 & 00.2270 & Mittl. Bericht & 28,42 & 26.15 \\
\hline 1 & 20.0260 & OP einer Inguinalhernie $<$ 7 Jahren & 582,58 & 535.97 \\
\hline 1 & 35.0030 & Technische Grundleistung OP I & 179,65 & 165.28 \\
\hline TOTAL & & & 796.65 \\
\hline Differenz zu IV & & & 353.40 \\
\hline Differenz in Prozent & & & $-30,7 \%$
\end{tabular}

Tabelle 2

Einkommensverhältnisse bei Senkung des TPW 2002-2004-200X unter der Annahme fixbleibender Ausgaben.

\begin{tabular}{|c|c|c|c|}
\hline & 2004 & 2005 & $200 x$ \\
\hline TPW & 0,97 & 0,90 & 0,85 \\
\hline Ertrag bei 100 TP & 97,00 & 90,00 & 85,00 \\
\hline Abzug Fixkosten $65 \%$ & $-65,00$ & $-65,00$ & $-65,00$ \\
\hline Bruttoerlös & 32,00 & 25,00 & 20,00 \\
\hline Prozent & $100,0 \%$ & $78,1 \%$ & $62,5 \%$ \\
\hline Theoretisches Einkommen, Basis Fr. 150 000.- & 150000.- & 117187.50 & $93750 .-$ \\
\hline
\end{tabular}

kommen im Jahr 2002 aus [4], so ist für unser kleines Rechenbeispiel ein durchschnittliches steuerbares Jahreseinkommen von Fr. 150 000.im Jahr 2002 durchaus realistisch. Gehen wir weiter davon aus, dass die Betriebskosten einer noch nicht voll amortisierten Praxiseinrichtung etwa $65 \%$ des Umsatzes betragen, hat sich das Einkommen, ausgehend vom Zürcher Taxpunktwert im KK-Bereich von Fr. -.90 im Jahre 2002 im Jahr 2005 bereits auf Fr. 117187.50 reduziert.

Sollten die von der santésuisse geforderten Taxpunktwerte von Fr. -.85 wahr werden, bleiben noch Fr. 93 750.- übrig, das heisst 37,5\% weniger als 2002! Wir bewegen uns damit knapp unter dem durchschnittlichen Jahreseinkommen einer Schweizer Familie im Jahre 1999 von Fr. 105 372.- (Tab. 2) [5].

Aufgefangen werden solche Einbussen wenigstens teilweise durch die entsprechende Steuersenkung. Doch was uns als Trost freut, dürfte die Steuerämter und die AHV mehr belasten: Am Beispiel der direkten Bundessteuer [6] und der AHV sind die Ertragsminderungen doch eindrücklich: Es fehlten allein in diesem staatlichen Einnahmebereich rund 137 Mio. Franken, nicht gerechnet die kantonalen und Gemeindesteuern! (Tab. 3).

Natürlich dürfen Krankenkassenprämien letztlich nicht dazu dienen, die Staatskasse zu füllen. Und doch - der geneigte Leser möge meine stümperhaften volkswirtschaftlichen Rechnungen verzeihen -, sie illustrieren, was das Drehen an nur einem Rädchen bewirken kann, und vermutlich ohne dass die Prämien um nur einen Rappen sinken werden (siehe bisherige Prämienentwicklung!).

\section{Eventuell ist eine politisch unbequeme Wurzelbehandlung nötig?}

Der Leser wird enttäuscht sein, weil er nun aufgrund der mir zugänglichen, verschiedensten Statistiken ein neues Schwarz-Peter-Spiel erwartet. Gegeneinander auszuspielen, wer an den verschiedenen Miseren Schuld trägt, wer am meisten verdient/verdienen darf oder gar welche Institution zu den kostenintensivsten gehört, überlassen wir den involvierten Kreisen zur geflissentlichen publizistischen Vermarktung. Interessant wäre höchstens nachzuforschen, warum sich Teile der Ärzteschaft unter der Drohung, eine staatliche Taxpunktwertregelung wäre deren Todesurteil, durch ein Ja zu TARMED das eigene Grab und dasjenige anderer geschaufelt haben ...

Auf jeden Fall halte ich den jetzigen Trend, vor allem durch TPW-Senkungen, aber auch die Aufhebung des Kontrahierungszwangs, staat- 
Tabelle 3

Theoretische Ertragsausfälle der direkten Bundessteuer und der AHV bei gesamtschweizerischer TPW-Senkung um 10 Rappen und konsekutiver Reduktion des steuerbaren Einkommens bei z.B. 15000 praktizierenden Ärztinnen/Ärzten [6].

OP-Tag inkl. Nebenkosten.

\section{Steuerbares Einkommen in Fr.}

2002: 120000 bis 149900

Zukunft?: 75000 bis 99900

Durchschnittliche AHV-Abgaben 9,5\%

2002: 120000 bis 149900

Zukunft?: 75000 bis 99900
Durchschnittlicher Steuerbetrag

$6724 .-$

2083.-

12825.-

8312.50
Total

$100860000 .-$

31245 000.-

-69615000 .

192375000 .-

124687500 .-

$-67687500 .-$

-137302500 .
Total Einbussen fung um die 59 Milliarden Franken, erwirtschaftet unter anderem mit 525000 Vollzeitstellen oder 17\% der Gesamtbeschäftigung [8]!

Am Aufzeigen der Friktionspunkte, an Vorschlägen aus der Basis fehlt es nicht: Ist TARMED wirklich schon veraltet [9]? Sollten wir nicht endlich ein gut geplantes, neues Finanzierungsmodell riskieren [10]? Dies sind nur zwei Beispiele aus jüngster Zeit.

Sparen - ja. Aber nicht nur auf dem Buckel der Leistungserbringer und Durchführungsstellen (früher «Arzt» genannt). Es mutet eben doch seltsam an, wenn das Bundesamt für Statistik aufzeigt, dass die indexierten Praxiskosten vom Index 100 im Jahre 1995 auf 100,9 im Jahre 2005, die Kosten der Spitäler hingegen auf 117,5 angestiegen sind, die Praxisindizes also immer etwa gleichbleibend vor und nach TARMED (!) [11]. Die Taxpunktwertsenkungen im Praxisbereich lassen vermuten, dass man den Sack schlägt, obwohl man den Esel meint ...

Kantonale, föderalistische Interessen, Angst vor Imageverlust an den Kliniken, Personalunionen in der Politik, Industrie und im Versicherungswesen, Verflechtungen in Verwaltungsräten, nicht zuletzt die Verschleuderung von Prämiengeldern für teure Werbeaktionen dürften allerdings schwer zu beeinflussen sein ...

\section{Literatur}

1 Merkblatt 2.02, www.avs.admin.ch $\rightarrow$ Merkblätter.

2 check up Nr. 1, 1.1.2000, S. 4, Eine unendliche Geschichte.

3 Stoffel U, Favrod-Coune C-A. Messphase der Kostenneutralität bei den FP-Ärzten erfolgreich abgeschlossen. Schweiz Ärztezeitung. 2005,86(25): 1491-2. Tabelle 1.

4 Hasler N. Einkommensverhältnisse der freien Ärzteschaft der Schweiz in den Jahren 2001 und 2002 Schweiz Ärztezeitung. 2006;87(3):87-93.

5 BFS. Einkommen, Lebensbedingungen und Konsum. Medienmitteilung. Neuenburg: BFS; 10. November 2005.

6 Finanzpolitik von A-Z, Januar 2004, www.efd. admin.ch.

7 Doser M, Egger Halbeis C, Schüpfer G. Die SwissDRG stellen neue Herausforderungen an das OP-Management. Schweiz Ärztezeitung. 2006;87(31/32):1371-6.

8 Zuccolini S. Das schweizerische Gesundheitswesen ist mehr als nur Kostenverursacher. Ars Medici. 2006; $15: 708$

9 Bossard F. TARMED veraltet? Braucht es eine Revision? Schweiz Ärztezeitung. 2005;86(10):614-8.

10 Canonica M. Ausstieg aus dem heutigen KVG anstelle der häppchenweisen Symptomtherapie. Schweiz Ärztezeitung. 2005;86(9):555-6.

11 Das Gesundheitswesen in der Schweiz. Ausgabe 2006,53 . sundheitssystems: 2002 betrug die Wertschöp- 